\title{
Right Atrium Mass in a 28-Year-Old Patient with Pulmonary Embolism Taking Contraceptives
}

\author{
Luis Jara-Palomares ${ }^{1}$, Maria Pilar Serrano-Gotarredona ${ }^{2}$, Jose Lopez- Haldón ${ }^{3}$, Maria José Rodriguez-Puras ${ }^{3}$, \\ Ignacio Bibiloni-Lage ${ }^{4}$, Elyan Ruiz-Solano ${ }^{4}$ and Remedios Otero-Candelera ${ }^{1}$
}

\author{
${ }^{1}$ Medical-Surgical Unit of Respiratory Diseases, University Hospital Virgen del Rocio, Seville, Spain \\ ${ }^{2}$ Radiology Unit, University Hospital Virgen del Rocio, Seville, Spain \\ ${ }^{3}$ Heart Area, University Hospital Virgen del Rocio, Seville, Spain \\ ${ }^{4}$ Cardiac Surgery Unit, University Hospital Virgen del Rocio, Seville, Spain
}

\begin{abstract}
We report the case of a 28-year-old woman taking contraceptives diagnosed with pulmonary embolism with a mass in the right atrium demonstrated by trans-thoracic echocardiogram that was not recognized on a previous angio-CT. Initially, it was thought to be a thrombus, but trans-oesophageal echocardiography and cardiac MRI showed data suggestive of cardiac neoplasm. Pericardial effusion and adjacent myocardial wall thickening noted on trans-oesophageal echocardiography were reported as signs that supported the possibility of malignancy, although cardiac MRI did not show wall infiltration signs. On the contrary, it demonstrated enhancement, which excludes the thrombotic nature of the mass and supports the diagnosis of neoplasm. The patient underwent surgery and biopsy proved that the mass was a myxoma. While myxomas are the most common among primary cardiac tumors, its attachment to the atrium free wall, far from the inter-atrium septum, the bi-lobed shape and accompanying pericardial effusion were atypical.
\end{abstract}

J Atheroscler Thromb, 2011; 18:829-832.

Key words; Pulmonary embolism, Cardiac mass, Myxoma, Heart neoplasm

\section{Introduction}

Myxomas comprise about $50 \%$ of benign cardiac tumours, and occur more frequently in left atrium. Pulmonary embolism (PE) is a rare complication of right atrium myxomas ${ }^{1)}$. Myxomas are often undiagnosed and pulmonary embolism originating from them is potentially fatal ${ }^{2,3)}$, so that in the absence of risk factors or clinical evidence of thrombosis, non-thrombotic pulmonary embolism should be suspected and echocardiography is recommended ${ }^{3,4)}$.

We present the case of a young woman that suffered a PE while taking contraceptives, a potential risk factor that could explain it. Cardiac imaging inciden-

Address for correspondence: Luis Jara-Palomares, MedicalSurgical Unit of Respiratory Diseases, University Hospital Virgen del Rocio, Afrodita n. 12, Bloque 2 Escalera 4, Piso 7, Número 1, 41014, Seville, Spain

E-mail: luisoneumo@hotmail.com

Received: March 9, 2011

Accepted for publication: April 7, 2011 tally discovered a right atrium tumor difficult to categorize, which was finally diagnosed as right atrium myxoma after a pathological test.

\section{Case Presentation}

A previously healthy 28-year-old woman complained of a cough, right chest pleuritic pain and dyspnea during the four days prior to admission complicated with haemoptysis (15-20 sputum days). She had neither fever nor thrombophlebitis symptoms, but she smoked 20 cigarettes per day and had been taking oral contraceptives for three months. Decreased breath sounds at the right base were highlighted on physical examination. Extremities showed no edema or signs of deep vein thrombosis (DVT). Chest X-ray revealed right pleural effusion and electrocardiogram showed a sinus rhythm of 72 beats per minutes with negative $T$ waves in III, aVF, V1-V4. Pulmonary embolism (PE) was suspected and a CT scan was requested. Pulmonary computed tomography angiogram (angio-CT) 
diagnosed PE accompanied by middle lobe parenchyma consolidation, consistent with infarct or hemorrhagic edema, and moderate pericardial effusion. Lower limb ultrasonography was normal. Given these findings we requested a trans-thoracic echocardiogram, which showed a mass in the right atria (visible but not recognized on angio-CT) and pericardial effusion, without signs of hemodynamic compromise. Full blood analyses with serum tumor markers were normal. Based on these data, it was suspected that there might be thrombus inside the right atrium. Transesophageal echocardiography and cardiac-MRI were requested to delineate the possible extension of the theoretical thrombus outside the atrium and further characterization of the lesion to establish, where appropriate, an alternative diagnosis to neoplasm. Trans-esophageal echocardiography (TEE) (Fig. 1) showed a large mobile mass within the right atrium with two lobes $(3.2 \times 16 \mathrm{~cm}$ and $3 \times 1.1 \mathrm{~cm})$, attached by a pedicle to the anterolateral wall of the atrium in the appendage area. Acoustic shadowing in the pedicle supported the presence of calcium. The anterolateral wall of the right atrium appeared thickened $(6.3 \mathrm{~mm})$. There was pericardial effusion $15 \mathrm{~mm}$ in width. Cardiac MRI (Fig. 2) demonstrated tissue formation inside the right atrium, similar to that described in TEE, composed of a pedicle attached to the right atrium appendage area with two mobile extensions and no signs of infiltration or invasion of cardiac structures.

Both the calcified pedicle and pericardial effusion described in TEE and contrast enhancement in cardiac MRI supported the suspicion of a neoplasm. Pericardial effusion and adjacent myocardial wall thickening noted on trans-esophageal echocardiography supported the suggestion of malignancy, although cardiacMRI did not show wall infiltration or thickening. Abdominal CT was normal. The mass was recognized in a retrospective review of chest CT angiography that initially diagnosed PE. Beam-hardening artefacts caused by the contrast column that enters the atrium from the superior vena cava could have hindered the identification of the mass (Fig. 2).

The surgical approach was through a medial sternotomy. Special care was taken regarding manipulation of the right atrium to avoid a new embolism. The right atrium was opened and a tumor of firm consistency, measuring $45 \times 25 \mathrm{~mm}$, adhering to the right atrium wall was removed. The atrium wall and the inflow tract of the right ventricle showed significant thickening. The histopathological examination confirmed the tumor etiology as a myxoma with extensive calcification and necrosis.

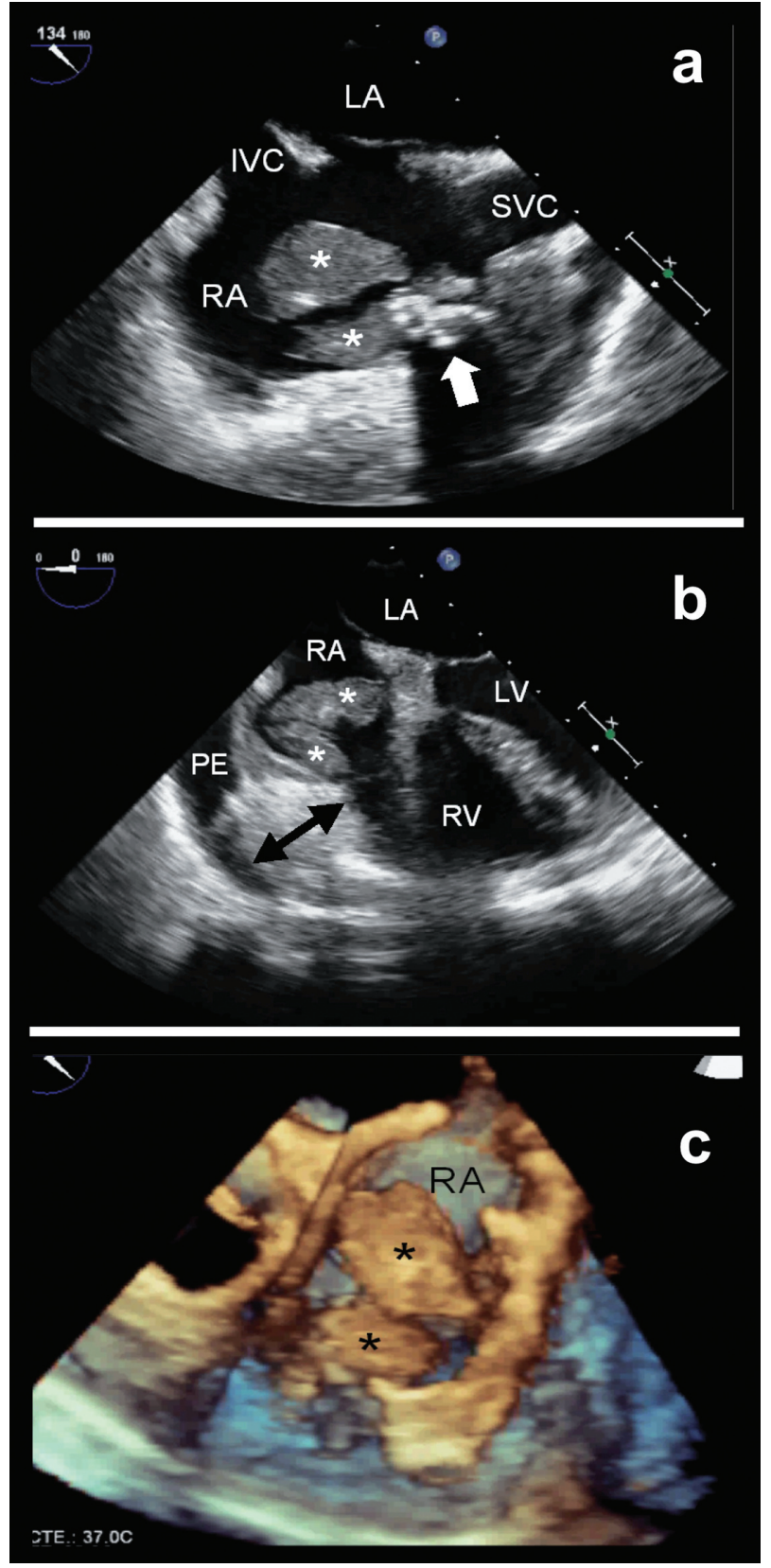

Fig. 1. (a) Image of two-dimensional trans-esophageal echocardiography in $134^{\circ}$ plane. We observe a mass composed of 2 lobes $(*)$ inserted through a pedicle on the anterior face of the right atrium (RA). The pedicle shows intense patchy calcification with acoustic shadowing (arrow). (b) Two-dimensional trans-esophageal echocardiography in transverse plane $\left(0^{\circ}\right)$ at mediumesophageal level. Shows the bi-lobed mass $\left(^{*}\right)$ within the right atrium (RA). The right ventricular (RV) wall near the atrioventricular groove is thickened (arrow) and pericardial effusion (PE) is seen. (c) Three-dimensional trans-esophageal echocardiography. We noted 2 lobes of the mass $\left(^{*}\right)$ within the right atrium. LV: Left ventricular; LA: Left atrium. IVC: Inferior vena cava; LA: Left atrium; SVC: Superior vena cava. 

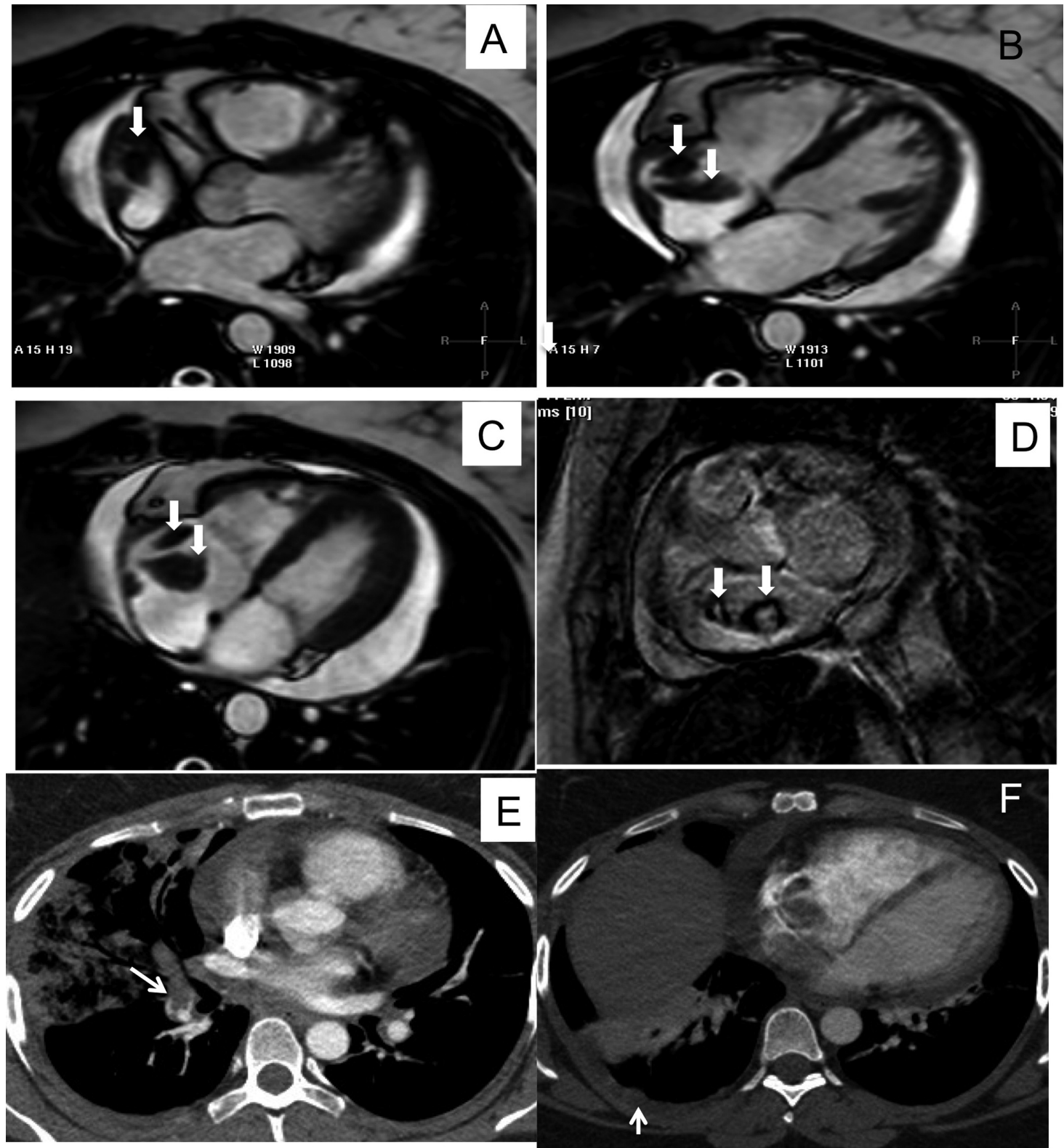

Fig. 2. (A), (B) and (C): Three contiguous axial images from a cine-bright blood acquisition (movement not shown) with balanced turbo field echo technique during a cardiac MRI show an bi-lobed mass (arrows) inside the right atrium (RA), which exhibits signal intensity similar to that of the myocardium and is attached by a pedicle to the anterior wall of the atrium in the area of the appendage. (D): Short axis plane almost sagittal to B and D, which demonstrates enhancement in the central core of the components of the mass after gadolinium injection, (E): Axial images from CT examination show intraluminal repletion defect in the intermedialis artery extending to the right middle artery (arrow in E). Image with parenchyma lung window settings (not included) better shows consolidation consistent with hemorrhagic edema. (F): Axial slice at almost the same level as $\mathrm{C}$ shows an intracavitary repletion defect with similar morphology. All images show free pericardial effusion (PE) and small pleural effusion is seen in $\mathrm{F}$ (arrow).

\section{Discussion}

Primary tumors are much less common than metastasis among cardiac neoplasm, and about 50\% are myxomas, most of them in the left atrium ${ }^{5)}$ and only about $15 \%$ arising in the right atrium, in which 
case they are usually attached to the inter-atrium septum. Therefore, in general, in a patient with cardiac neoplasm and no evidence of extra cardiac tumor, myxoma is the most probable diagnosis ${ }^{6-8}$. Pulmonary embolism and pulmonary hypertension are co-morbidities of right atrium myxoma ${ }^{9,10)}$. Besides the possible disturbance of heart activity due to valve obstruction, bleeding and fragmentation can also occur with subsequent embolism ${ }^{11)}$. This seems more likely to happen in the left heart, probably because higher pressure facilitates tumor fragmentation ${ }^{12)}$. About 20 similar cases of pulmonary embolism due to myxoma have been reported ${ }^{4)}$. Patients are often released undiagnosed and pulmonary embolism is potentially fatal, so that in the absence of risk factors or clinical evidence of thrombosis, non-thrombotic pulmonary embolism should be suspected and echocardiography is recommended $^{2-4)}$. In our patient, risk factors were present so the pulmonary embolism could be explained, but incidental echocardiography revealed the presence of the mass. Multi-slice CT also revealed a mass, although it went unnoticed. This should encourage radiologists to look inside the heart when interpreting a CT pulmonary angiography that reveals pulmonary embolism. Beam-hardening artefacts from the column of contrast entering the atrium from the vena cava could explain this, and it could have been partly obviated by softening the lung window settings. The main differential diagnosis should be free-floating thrombus in transit. Neither echocardiography nor CT is specific enough to distinguish between them. Enhancement on cardiac MRI could be considered definitive to distinguish the mass from a thrombus in our case. Heterogeneous enhancement is typical of myxomas as well as the presence of thrombus on the surface and calcification within the mass $\left.{ }^{13}{ }^{14}\right)$. The last two, confirmed on histopathological examination, could explain the absence of enhancement in the periphery (Fig. 1D) and in its pedicle.

Its location in the free wall of the right atrium, the bi-lobed shape and accompanying pericardial effusion represent an atypical form of presentation.

\section{References}

1) Wun T, White RH: Epidemiology of cancer-related venous thromboembolism. Best Pract Res Clin Haematol, 2009; 22: $9-23$

2) De Carli E, Sechi LA, Ciani R, Barillari G, Dolcetti G, Bartoli E: Right atrial myxoma with pulmonary embolism. Cardiology, 1994; 84: 368-372

3) Battellini R, Bossert T, Areta M, Navia D: Successful surgical treatment of a right atrial myxoma complicated by pulmonary embolism. Interact CardioVasc Thorac Surg, 2003; 2: 555-557

4) Jardine DL, Lamont DL: Right atrial myxoma mistaken for recurrent pulmonary thromboembolism. Heart, 1997; 78: 512-514

5) Burke A, Virmani R: Tumors of the heart and great vessels. Atlas of Tumor Pathology. 3rd ed. Washington DC: Armed Forces Institute of pathology; 1996

6) Goswami KC, Shrivastava S, Bahl VK, Saxena A, Manchanda SC, Wasir HS: Cardiac myxomas: clinical and echocardiographic profile. Int J Cardiol, 1998; 63: 251-259

7) McCoskey EH, Mehta JB, Krishnan K, Roy TM: Right atrial myxoma with extracardiac manifestations. Chest, 2000; 118: 547-549

8) Riise GC, Bugge M, Johnsson AA, Willen H: A 40- yearold male with cough, hemoptysis, and increasing dyspnoea. Eur Respir J, 2001; 18: 432-435

9) Case records of the Massachusetts General Hospital. Weekly clinicopathological exercises. Case 17-1993. A 53-year-old woman who died after several years of a dementing illness with intermittent generalized seizures and abnormal movements of the extremities and head. $\mathrm{N}$ Engl J Med, 1993; 328: 1259-1266

10) Reynen K: Cardiac myxomas. N Engl J Med, 1995; 333: 1610-1617

11) Fracasso T, Varchmin-Schultheiss K: Sudden death due to pulmonary embolism from right atrial myxoma. Int J Legal Med, 2009; 123: 157-159

12) Virmani R, Posey DM, Clark MA, McAllister HA: Right atrial myxoma causing pulmonary emboli and pulmonary hypertension. Am J Forensic Med Pathol, 1982; 3: 249-252

13) Mollet NR, Dymarkowski S, Volders W, Wathiong J, Herbots L, Rademakers FE, Bogaert J: Visualization of ventricular thrombi with contrast-enhanced magnetic resonance imaging in patients with ischemic heart disease. Circulation, 2002; 106: 2873-2876

14) Masui T, Takahashi M, Miura K, Naito M, Tawarahara K: Cardiac myxoma: identification of intratumoral hemorrhage and calcification on MR images. Am J Roentgenol, 1995; 164: 850-852 